\title{
Le donne di María Zambrano. L'attualità del suo pensiero in una prospettiva filosofico-femminile.
}

\author{
The Women of Maria Zambrano: \\ The Timeliness of Her Thought From \\ a Female Philosophical Perspective
}

Sara del Bello Università degli Studi di Roma la Sapienza sara.delbello.x@gmail.com

DOI: https://doi.org/10.15366/bp2020.25.011

Bajo Palabra. II Época. No25. Pgs: 239-250 


\section{Resumen}

La atención que María Zambrano presta al universo femenino se concreta tanto a través de páginas de intensa reflexión sociopolítica, como gracias a una investigación linguístico-conceptual de la que sus mujeres son expresión viva. Tocando, en particular, el mundo de Eloísa y Antígona, en este breve espacio, tenemos la oportunidad de desarrollar los temas de la responsabilidad, de la ética y de la conciencia a través de un análisis político, que es una característica clave de su universo, también cuando no se manifiesta explícitamente. Y una vez más, la filósofa española deja que emerjan sus rasgos distintivos, a menudo precursores de muchos temas posteriores, como el pensamiento de la diferencia sexual.

Palabras clave: mujeres, Antígona, feminismo, Eloisa, conciencia, responsabilidad, razón, ética.

\section{Abstract}

The attention María Zambrano pays to the female universe takes shape both through pages of intense socio-political reflection and through a linguistic-conceptual research of which women are a living expression. Touching the world of Eloisa and Antigone in particular within this brief space, we have the opportunity to develop the topics of responsibility, ethics, and conscience through a political analysis; a key feature in the Zambranian universe, even when it does not appear explicitly. Yet once again, the Spanish philosopher lets her distinguishing features emerge, often a forerunners of many themes, such as the thinking of sexual differences.

Keywords: women, Antigone, feminism, Eloisa, conscience, responsibility, reason, ethics. 


\section{Una breve introduzione}

LA PRIMA METÀ del secolo scorso racchiude un insieme di voci intellettuali che non ci è possibile etichettare come femminista ma che, al tempo stesso, contribuisce allo sviluppo di idee, forme e strutture che rompono alcuni schemi di un pensiero universalizzante e monolitico. Autrici quali Hannah Arendt, Simone Weil, Edith Stein, María Zambrano esplorano, infatti, differenti modalità filosofiche in cui, non solo si lasciano le tracce di un successivo pensiero femminista, ma si enucleano tratti e caratteristiche che ci permettono di parlare di un filosofare al femminile.

È la stessa Zambrano a ricorrere a quest'ultimo aggettivo per raccontare se stessa: non fu, infatti, femminista, bensì femminile. Da sempre ostile ad ogni tipo di definizione che potesse, in quanto tale, limitare e stabilire dei confini piuttosto che lasciare aperto il pensare, la filosofa andalusa sceglie di dare forma al suo essere donna ed intellettuale grazie alla costante ricerca di un linguaggio e di un universo speculativo capaci di esprimere le differenze e l'eterogeneità proprie dell'esistenza.

Non è, dunque, casuale la scelta di figure femminili nell'ambito del suo panorama filosofico. Alle sue donne lascia, così, il compito di plasmare la sua stessa riflessione. Lo faranno, ad esempio, la giovane Eloisa, la serva Nina, la sacerdotessa Diotima, la mistica Santa Teresa. Ma più di tutte sarà Antigone a farsi portavoce di un pensiero della differenza sessuale, di un dialogo etico-politico, di un gesto pietoso ed accogliente.

Lepitome della sua visione filosofico-politica, la coscienza nascosta negli abissi dell'interiorità umana, l'aurora mai venuta alla luce, il simbolo di un esilio politico, ma anche ontologico ed esistenziale: questo il ruolo assegnato all'eroina greca nella rivisitazione zambraniana della tragedia sofoclea.

Le sue donne, quelle che prendono la parola nei suoi scritti, sono le donne della sua Spagna, della sua Europa, cui la società del tempo nega l'espressione del proprio pensiero, la possibilità di accedere allo spazio pubblico e di varcare il confine delle mura domestiche.

\section{Il ruolo della donna}

CosA SIGNifichi essere donna in quel determinato momento storico-politico accompagna María Zambrano sin dalle sue prime scelte di vita. I suoi racconti auto- 
biografici ne costituiscono una chiara testimonianza là dove confessa di aver, fin da piccola, immaginato di coronare il suo sogno di diventare cavaliere templare, senza per questo rinunciare alla sua condizione femminile.

Lautrice dedica alcuni scritti giovanili, risalenti al 1928, all'analisi del rapporto donna-uomo muovendo dalla constatazione della condizione femminile del suo tempo: "[...] tra le tante differenze tra maschio e femmina [...], la più evidente consiste in ciò che la donna dice e in ciò che tace, soprattutto se pensiamo a quanto la donna sia stata condannata al silenzio e abbia dovuto condurre la propria vita a forza di tacere". ${ }^{1}$

Sviluppa, così, un'attenta analisi della donna europea e in particolare, spagnola, essenzialmente consacrata dalla storia al focolare domestico, privata dunque della possibilità di esercitare il giudizio pubblico, di manifestare il proprio consenso o dissenso politico, di prendere parte in modo attivo alla vita sociale del proprio Paese.

Per Zambrano è, invece, arrivato il momento di contribuire al cambiamento della Spagna di quegli anni grazie alla partecipazione comune di donne e uomini, della nuova e della vecchia generazione, che dimostrino il proprio impegno nel ricostruire le fondamenta di una società ormai anacronistica.

L'appello, per così dire, che la filosofa andalusa rivolge alle donne della sua epoca è di dare voce alla Spagna, ad una terra che necessita di abbandonare una forma -quella monarchica- non più adatta a rappresentarne bisogni e necessità: "la Spagna non aveva motivo di tenersi questo re per potersi riconoscere nell'immagine che le offriva" ${ }^{2}$ e soprattutto, era a suo giudizio indispensabile promuovere quel processo di avanzamento della democrazia che, proprio in quanto tale, presupponeva l'incontro molteplice di punti di vista differenti per "respirare liberamente, profondamente, all'unisono [...] il ritmo di un respiro comune". ${ }^{3}$

Nel costruire la sua lucida riflessione, Zambrano pone l'accento su alcuni elementi, essenziali per comprendere appieno l'apporto da lei fornito alla filosofia femminile contemporanea.

In primo luogo, il suo rifiuto di una definizione femminista del proprio pensiero deriva dalla critica rivolta all'appiattimento delle rivendicazioni femministe ad una mera richiesta di eguaglianza giuridica tra uomini e donne, tale da ridurre il tutto ad una condizione di parità, intesa come pericolosa omologazione:

perché nessuno può pensare che la donna abbia colmato la sua ansia liberatrice con la cosiddetta 'emancipazione economica'. [...] L'ideale femminista, per usare il vecchio termine,

\footnotetext{
${ }^{1}$ Zambrano, M., Unamuno, Bruno Mondadori, Milano 2006, pp. 48-49.

2 Id., Delirio e destino, Raffaello Cortina editore, Milano 2000, p. 137.

${ }^{3}$ Ivi, p. 43.
} 
sta al di là della 'emancipazione economica', che non è altro che un primo passo tristemente necessario. ${ }^{4}$

In altri termini, per María Zambrano, è indispensabile che la donna dia forma alle proprie differenze in vista di un'integrazione spirituale con gli uomini. La filosofa spagnola si fa portavoce, in questo senso, di un pensiero della differenza sessuale capace, cioè, di costruire uno spazio in cui il femminile possa trovare la propria specifica modalità di esprimersi, senza mai dover rinunciare alle proprie peculiarità sacrificandosi in nome di un paventato soggetto universale.

Ecco, dunque, che gli articoli zambraniani pubblicati sulla rivista El Liberal, nella sezione Mujeres, dal marcato accento civile e politico e fortemente incentrati sul ruolo della donna, chiedono tanto a quest'ultima, quanto alle nuove generazioni, di cui anche Zambrano è parte attiva, di riscattare se stessi e la propria patria, risvegliandola da un profondo torpore che, tra le altre cose, ha impedito - come lei stessa scrive- quel clamore appassionato che la donna europea ha, invece, conosciuto nel corso del XIX secolo:

La donna avanza nella sua evoluzione, [...] lotta e si sforza [...].

Di fronte a questo cambiamento femminile, l'uomo si impaurisce [...]. In alcuni uomini esaltati $[\ldots]$ la loro dignità da gallo non può permettere che la donna $[\ldots]$ non esaurisca la sua esistenza al servizio dei loro destini.

[...] Ci viene in mente solo un rimedio per questo: comunità di ideali, integrazione spirituale delle loro vite. ${ }^{5}$

Per l'intellettuale andalusa è, quindi, importante che le donne acquisiscano piena consapevolezza della propria femminilità e prendano coscienza della propria condizione socio-politica:

la ragazza di oggi è scossa da un irriducibile sconcerto di fronte allo spettacolo che si osserva per le vie della città, della schiavitù effettiva di una parte delle persone del suo stesso sesso. Sconcerta $[\ldots]$ il fatto che migliaia di donne siano ancora considerate alla stregua di cose o di oggetti, che la loro femminilità sia sfruttata con il consenso della legge.

[...] L'energia che le nostre signorine del xix secolo [...] non hanno saputo trasformare in urlo, in grido, in agitazione esaltata, dobbiamo averla noi, le ragazze di questo 'frivolo Ventesimo secolo', trasformata, capovolta, produttiva $[\ldots]$ in una lotta decisa e forte $[\ldots]{ }^{6}$

\footnotetext{
${ }^{4}$ Id., Donne, Morcelliana, Brescia 2006, p. 56.

${ }^{5}$ Ivi, pp. 73-74.

${ }^{6}$ Ivi, pp. 52-53.
} 
In secondo luogo, nel plasmare queste sue considerazioni sul ruolo della donna, Zambrano muove evidentemente una critica nei confronti, non solo del contesto storico-culturale spagnolo, ma anche di un orizzonte filosofico più ampio, imperniato su quella ragione razionalista e assoluta, da lei costantemente messa in discussione nel corso di tutto il suo filosofare.

Senza addentrarci in questo aspetto - per il quale sarebbe opportuno sviluppare ulteriori considerazioni- ciò che risulta rilevante ai fini del nostro discorso consiste nel mettere in luce come l'apporto zambraniano al pensiero femminile prenda corpo lungo due direzioni: da un lato, le riflessioni più propriamente politiche, dall'altro, la ricerca di una modalità filosofica capace di contenere e accogliere l'universo femminile.

L'uomo europeo, come lei stessa sottolinea, ha sempre vissuto mosso da un intento volontaristico, il cui esito si è risolto in quell'assolutismo - da intendersi nell'accezione tipicamente zambraniana del termine - vale a dire in quell'irrefrenabile ansia dominatrice, volta a inglobare tutto al di sotto di sé, perdendo di vista il particolare, il differente; in un soggettivismo estremo che ha condotto ad un'Europa agonizzante, vittima di una ragione fredda e calcolatrice, incapace di entrare in relazione con l'altro.

La donna, invece, esprime un sentire originario, a contatto con l'elemento vitale, con l'aspetto viscerale dell'esistenza. Si tratta non di un atteggiamento irrazionale, bensì di una differente modalità di intendere la ragione, là dove quest'ultima non consiste più in quel mero strumento di controllo totalizzante della realtà, ma in una ragione poetica e materna, creatrice e pietosa, fiduciosa in ciò che è dato e ricevuto, disposta, quindi, ad aprirsi anche verso ciò che rimane nell'ombra e che "nel suo oscuro palpitare crea chiarezza". ${ }^{7}$

Zambrano parla più precisamente di un saber del alma, simbolo ed espressione di un percorso di decostruzione di quel soggetto monolitico ed autoreferenziale di cui si è detto in precedenza. E il sapere dell'anima, capace di comprendere la molteplice eterogeneità del reale, è ciò di cui le sue figure femminili sono rivelazione.

\section{Oltre l'universalismo maschile}

Questo idealista uomo occidentale vive della volontà [...]. È un idealismo volontaristico e attivista che sogna di sottomettere la realtà intera alla propria orbita. È la radice guerriera di tutta la cultura occidentale. E [...] nel suo idealismo l'uomo inventa anche la donna [...].

7 Id., Chiari del bosco, Bruno Mondadori, Milano 2004, p. 63 
La donna è una donna che corrisponde all'idea platonica, che come lei è assoluta, cioè pura e come lei fa da mediatrice, da intermediaria.

[...] La donna è qui dipendente in tutto, in un sistema di totale obbedienza. È l'uomo che l'ha creata $[\ldots]$; lei è parte integrante della creazione maschile $[\ldots]{ }^{8}$

Queste parole costituiscono la premessa ad una importante figura del panorama filosofico zambraniano, appartenente alla cultura medievale: Eloisa.

Nella visione di María Zambrano, la fanciulla innamorata di Abelardo assume un particolare significato poiché incarna un simbolo di indipendenza, intesa non come mancanza di legame, bensì come accoglimento pieno del proprio amore, nato dalla consapevolezza e dalla coscienza di sé e dell'essere in relazione all'altro.

È attraverso la propria passione che Eloisa compie una scelta che, nell'interpretazione della filosofa spagnola, la distanzia da quei modelli di stampo maschile, tipici del Medioevo, tali da ingabbiare la donna in ruoli prefissati e soggiogati, ossia la moglie e la donna angelica.

Per Zambrano, invece, solo la cortigiana, da un lato e la monaca, dall'altro, scardinano queste forme di controllo nei confronti della figura femminile del tempo. Entrambe, infatti, obbediscono primariamente a se stesse, sebbene ciascuna in modo diverso.

Nel caso di Eloisa, "il rifiuto di contrarre le nozze e la scelta di seguire l'amato nel suo destino, che la porterà ad essere rinchiusa tra le mura di un convento, rispondono alla volontà di vivere fino in fondo la propria scelta d'amore". ${ }^{9}$

In altri termini, Zambrano sottolinea come la giovane Eloisa metta, in qualche modo, in discussione una concettualizzazione della donna - docile, asservita e pura- espressione non di se stessa, ma proiezione delle fantasie maschili dell'epoca, tali da renderla niente più che un oggetto dei propri desideri, facilmente controllabile e manipolabile da parte dell'uomo.

Ma ancor più di Eloisa, sarà Antigone - la celebre protagonista della tragedia greca, reinterpretata dalla pensatrice spagnola- ad assumere su di sé il significato più profondamente politico della filosofia zambraniana.

\footnotetext{
${ }^{8}$ Id., All'ombra del Dio sconosciuto. Antigone, Eloisa, Diotima, Nuova Pratiche Editrice, Milano 1997, pp. 67-69.

9 Del Bello, S., Esperienza, politica e antropologia in María Zambrano. La centralità della persona, Mimesis, Milano 2017, pp. 113-114.
} 


\section{La coscienza sepolta viva.}

"L’Antigone zambraniana sarà simbolo di una coscienza aurorale, in grado di illuminare il destino degli uomini e delle donne della sua stirpe, pur collocandosi nello spazio delle tenebre e degli inferi, al riparo dalla luce del giorno". ${ }^{10}$

Oggetto di numerose attenzioni, questo personaggio femminile ha attirato su di sé lo sguardo di tanti intellettuali nel corso della storia, subendo molteplici interpretazioni, non ultima quella della filosofa andalusa:

Antigone, in verità —scrive Zambrano- non si suicidò nella sua tomba [...]. E come poteva [...] darsi la morte, lei che non aveva mai disposto della sua vita? [...]

Il conflitto tragico la trovò vergine e la prese interamente per sé [...]. Senza di lei, la tragica vicenda della sua famiglia e della città non avrebbe potuto avere un seguito.

[...] Il conflitto tragico, infatti, non arriverebbe a essere tale [...] se dalla distruzione non discendesse qualcosa che la oltrepassa, che la riscatta.

Quel qualcosa è appunto Antigone. Il compito assegnatole da Zambrano è di mediatrice, che si risolve nel mettere in dialogo i vivi con i morti, le ombre con le luci, l'interiorità con l'esteriorità.

La fanciulla compie, così, un viaggio di iniziazione, dove ricercandosi finisce per ritrovare non solo i fili ingarbugliati della sua giovane esistenza, ma anche quelli della sua città e con essi, della sua famiglia e della sua tragica storia.

Nasce una seconda volta - espressione cara alla filosofa spagnola- poiché nella tomba, in cui viene costretta per decisione di Creonte, ha la possibilità di scoprire finalmente chi sia, di guardarsi pienamente, come mai prima di allora, di scendere nelle profondità della propria coscienza. Il viaggiare al di sotto del suolo è la metafora di un cammino all'interno di se stessa e il discendere altro non è che il simbolo di una salita. Arrivare alla coscienza di sé -chiarità nata dagli abissi- passa attraverso l'esposizione alla vista altrui: colui che nasce è prima di tutto un cieco - afferma Zambrano- ed è quindi oggetto di sguardi, di giudizio, di attenzione. L'essere sottoposti, nudi e inermi, alla visione dell'altro è il primo atto che segna la venuta al mondo, l'essere cioè privi di quella condizione protettiva propria, invece, dell'utero materno, in cui si è al riparo da tutto e da tutti.

L'offrirsi agli occhi di chi ci è di fronte è, dunque, il gesto cui si abbandona Antigone, scegliendo di confrontarsi con le anime di coloro che l'hanno accom-

${ }_{10}$ Ivi, p. 86. 
pagnata in vita, al di fuori della caverna. È, quindi, muovendo dal riconoscimento dell'altro e dall'ascolto di un differente punto di vista che nasce e prende forma il dialogo, su cui si gettano le basi di una dimensione sociale costruttiva, relazionale e democratica.

L'eroina greca è il simbolo della visione politica zambraniana. Offre, infatti, una prospettiva diametralmente opposta ad una concezione individualista ed autoreferenziale, pienamente incarnata dal personaggio edipico, su cui più volte la filosofa spagnola si sofferma nelle sue opere. Ai perfetti antipodi di Antigone, il padre della giovane fanciulla soffre del timore di essere visto, aspirando al tempo stesso ad una visione totale e completa della realtà. In altri termini, si sottrae all'incontro con l'altro, il che lo conduce all'impossibilità di vedere se stesso: per questo finisce vittima del suo ambizioso sogno di potere o - per riprendere il linguaggio zambraniano- rimane attaccato alla sua placenta oscura, costantemente accompagnato, da un lato, da un'irrefrenabile ansia di dominio e, dall'altro, dall'incapacità di esistere nascendo. Edipo è mosso dal desiderio "di dover essere saggio immerso nella cecità; di dover scoprire la natura delle cose, senza nemmeno conoscere se stesso". ${ }^{11}$

Antigone incarna, invece, i valori fondanti l'idea di democrazia; è, infatti, l'espressione di un percorso auto-coscienziale, presupposto indispensabile ad una successiva dinamica relazionale e di un modello di responsabilità, al tempo stesso, individuale e collettiva.

Grazie alle sue capacità di ascolto e attenzione, i personaggi che dialogano con la giovane protagonista all'interno della caverna oscura hanno modo di arrivare a comprendere più pienamente se stessi:

sembra che questa, di dover discendere negli abissi, sia la condizione per poter ascendere attraversando tutte le regioni in cui l'amore è l'elemento, per così dire, della trascendenza umana: originariamente fecondo, quindi, se persiste, creatore. Creatore di vita, di luce, di coscienza.

È l'amore, infatti, con questo suo rituale viaggio agli inferi, a illuminare la nascita della coscienza. ${ }^{12}$

L'amore, la trascendenza, la pietà, la nascita e la speranza - concetti cardine del pensiero zambraniano- sono perfettamente rappresentati da Antigone. Non ci è possibile approfondirli in questa sede, ${ }^{13}$ ma è doveroso metterli in luce nel descri-

${ }_{11}$ Zambrano, M., Il sogno creatore, Bruno Mondadori, Milano 2002, p. 106.

12 Id., La tomba di Antigone. Diotima di Mantinea, La Tartaruga Edizioni, Milano 2001, p. 46.

${ }^{13}$ All'interno del mio saggio - precedentemente citato- ho avuto modo di approfondire differenti concetti chiave della figura di Antigone, così come viene letta da María Zambrano. Oltre a quelli rapidamente menzionati nel 
vere questa figura femminile tanto rilevante nel suo universo speculativo, in quanto espressione della sua visione antropologica, a sua volta requisito fondamentale della sua concezione politica.

Se la persona costituisce l'essere che patisce la propria trascendenza e l'amore consiste nella forza costitutiva che dà ragione dell'umana relazionalità, in quanto "agente di trascendenza nell'uomo", ${ }^{14}$ allora Antigone ne è la perfetta incarnazione. Mossa dalla pietà, animata dalla speranza, chiamata a nascere costantemente, si spinge oltre il proprio sé per arrivare a cogliere il punto di comunanza perduta tra tutte le creature umane.

Nell'ambito di questo rapido excursus, vorrei infine porre l'accento su un ultimo aspetto, specifico del personaggio di Antigone e, come precisato, della filosofia di Zambrano: il concetto di pietà.

Il dominante pensiero razionalista - di cui si è detto in precedenza e che, nell'ottica zambraniana, si identifica in modo particolare con il razionalismo cartesiano e l'idealismo hegeliano- ha del tutto obliato il sentimento della pietà. E quest'ultima si risolve per Zambrano nella capacità di saper "trattare con l'altro [che] significa semplicemente trattare con la realtà". ${ }^{15}$ L'essere pietosi è l'atteggiamento di accettazione della realtà, di apertura nei confronti dell'alterità - anch'essa costitutiva del nostro essere umani- ed è la piena coscienza dell'ontologica limitatezza che ci contraddistingue in quanto creature finite e imperfette. Ben distante dalla tolleranza, la pietà insegna l'amore, il che vuol dire l'accoglimento dell'esistenza in tutti i suoi chiaroscuri, comprensivi di ciò che scorre nelle viscere e non si espone alla luce accecante di un rigido sapere razionalista. Significa, quindi, aprirsi alle singole unicità di cui consta la vita, superando una visione totalizzante e accentratrice, incapace di comprendere l'impossibilità di ridurre l'esistente ad una unità di identità.

\section{In conclusione}

La sua Antigone, e in generale, le sue donne - portatrici non solo del suo pensiero, ma anche della sua esperienza vitale - testimoniano le sue stesse scelte politiche, sociali ed esistenziali. Raccontano il suo percorso da esule, il suo cammino di donna

\footnotetext{
presente articolo, viene inoltre messo in luce un confronto rispetto all'interpretazione hegeliana dello stesso personaggio femminile, ponendo in risalto, in particolare, gli elementi della sorellanza e del materno, decisivi nell'analisi zambraniana. Cfr. Esperienza, politica e antropologia in Maria Zambrano, cit., pp. 105-137.

${ }_{14}$ Zambrano, M., L'uomo e il divino, Edizioni Lavoro, Roma 2009, p. 249.

15 Ivi, p. 188.
} 
in un'Europa - anche quando osservata in lontananza - segnata tragicamente dalla distruzione e dalla violenza più brutali.

Le sue figure femminili rappresentano, così, le idee di memoria e responsabilità. Soprattutto Antigone costituisce la viva espressione di colei che sceglie di assumere su di sé il peso delle colpe altrui, così da poter finalmente cogliere il senso di tanto dolore, riscattando - come detto- la vicenda storica della sua città.

Grazie ad Antigone, la storia conosce finalmente la possibilità di incamminarsi lungo la strada dell'etica, superando la sua logica tragica e sacrificale. La fanciulla greca dimostra, infatti, la volontà di prendersi cura del mondo di cui è parte -l'amor mundi di cui parla anche Arendt- quello stesso mondo che, per mano umana, ha finito per renderla esule.

Umanizzare la storia nasce, dunque, da un gesto di assunzione di responsabilità di fronte a ciò che si pone in essere o, come direbbe Zambrano, si traduce nel portare avanti la propria nascita continua "con la responsabilità di vedere e di essere visto, di giudicare e di essere giudicato, di dover edificare un mondo" ${ }^{16}$ in cui sia riconosciuta piena centralità ad ogni persona.

${ }^{16}$ Id., Il sogno creatore, cit., p. 27. 


\section{Referencias Bibliográficas}

Del Bello, S., Esperienza, politica e antropologia in María Zambrano. La centralità della persona, Mimesis, Milano 2017.

Del Bello, S., "Democrazia e demagogia a confronto. Brevi considerazioni su alcuni concetti chiave della visione politica zambraniana", in Aurora. Papeles del Seminario Maria Zambrano, Universitat de Barcelona, n. 19 (2019), pp. 32-37.

Del Bello, S., "Il genocidio ruandese del '94 e la difesa dell'umano nel pensiero filosofico di Zambrano, Arendt, Hillesum”, in B@belonline, RomaTrE-Press, n. 3 (2017), pp. 309-322.

Zambrano, M., Allombra del Dio sconosciuto. Antigone, Eloisa, Diotima, Nuova Pratiche Editrice, Milano 1997.

Zambrano, M., Chiari del bosco, Bruno Mondadori, Milano 2004.

Zambrano, M., Delirio e destino, Raffaello Cortina editore, Milano 2000.

Zambrano, M., Donne, Morcelliana, Brescia 2006.

Zambrano, M., Il sogno creatore, Bruno Mondadori, Milano 2002.

Zambrano, M., La tomba di Antigone. Diotima di Mantinea, La Tartaruga Edizioni, Milano 2001.

Zambrano, M., L'uomo e il divino, Edizioni Lavoro, Roma 2009.

Zambrano, M., Unamuno, Bruno Mondadori, Milano 2006.

DOI: https://doi.org/10.15366/bp2020.25.011

Bajo Palabra. II Época. No25. Pgs: 239-250 